\title{
Inclusive Lucky Labeling of Graphs
}

\section{${ }^{1}$ R. Bhuvaneswari, ${ }^{2}$ K. Bhuvaneswari}

\author{
${ }^{1}$ Assistant Professor \\ Post Graduate Department of Mathematics \\ BMS College for Women \\ Bengaluru, India \\ buviak@gmail.com \\ ${ }^{2}$ Assistant Professor \\ Post Graduate Department of Mathematics \\ BMS College for Women \\ Bengaluru, India \\ bhuvanaklu@gmail.com
}

Article History: Received: 11 January 2021; Accepted: 27 February 2021; Published online: 5 April 2021

\begin{abstract}
ILL) and study proper inclusive lucky labeling (PILL) for simple undirected graphs. We also define inclusive lucky number and proper inclusive lucky number for some simple graphs.
\end{abstract}

Keywords- Lucky labeling; proper lucky labeling; inclusive lucky labeling; proper inclusive lucky labeling; Inclusive lucky number; proper inclusive lucky number

\section{INTRODUCTION}

A graph labeling is an assignment of labels (or integers) to vertices or edges or both. A label is said to be proper if no two adjacent vertices have same label. The concept of labeling was introduced by Rosa [4] in 1967. It was further developed by Graham and Sloane in 1980 [3].

Several types of labeling were introduced and studied for different types of graphs. The concept of Lucky labeling of graphs were studied by A. Ahadi et al [1] and S. Akbari et al [2]. Lucky number for different kinds of graphs has been studied.

In this paper, we define a new notion of labeling called inclusive lucky labeling (ILL). We also define proper inclusive lucky labeling (PILL), inclusive lucky number and proper inclusive lucky number for some simple undirected graphs.

\section{PRELIMINARIES}

In this section, we provide some basic definitions and results as in $[1,2]$.

Let $G$ be a simple graph with vertex set $V(G)$. For any vertex $v$ in $G, N(v)$ denotes the set of all the vertices which are adjacent with $v$ and $N[v]=N(v) \mathrm{U}\{v\}$.

Definition 1. Let $f: V(G) \rightarrow\{1,2,3, \ldots\}$ be a labeling of the vertices of a graph $G$ and let $S(v)$ denote the sum of labels over the neighbors of the vertex $v$ in $G$. For an isolated vertex $v, S(v)=0$.

A labeling $f$ is said to be a lucky labeling or simply lucky if $S(u) \neq S(v)$ for every pair of adjacent vertices $u$ and $v$.

The least positive integer $k$ for which there exists a lucky labeling $f: V(G) \rightarrow\{1,2,3, \ldots, k\}$ for the graph $G$ is called lucky number of a graph $G$ and it is denoted by $\eta(G)$.

Definition 2. A labeling $f$ is said to be proper lucky labeling if $f$ is lucky and proper. The proper lucky number $\eta_{p}(G)$ of a graph $G$ is the least positive integer $k$ for which $\mathrm{G}$ has a proper lucky labeling with $\{1,2,3, \ldots, k\}$ as the set of labels.

Example: For complete graph $K_{n}, \eta\left(K_{n}\right)=2$ and $\eta_{p}\left(K_{n}\right)=4$.

Result: For any connected graph $\mathrm{G}, \eta(G) \leq \eta_{p}(G)$.

\section{INCLUSIVE LUCKY LABELING}

In this section, we define a new notion of labeling called inclusive lucky labeling and proper inclusive lucky labeling. We also define the inclusive lucky number and proper inclusive lucky number. Further, we discuss the inclusive lucky number and proper inclusive number of some standard graphs.

Definition Let $f: V(G) \rightarrow\{1,2,3, \ldots\}$ be a labeling of the vertices of a graph $G$ and let S[v] denote the sum of labels over the neighbors of the vertex $v$ in $G$ including $v$. For an isolated vertex $v$, put $\mathrm{S}[v]=1$ and $f(v)=1$. 
A labeling $\mathrm{f}$ is said to be inclusive lucky labeling if $S[u] \neq S[v]$ for every pair of adjacent vertices $u$ and $v$. The least positive integer $k$ for which there exists an inclusive lucky labeling $f: V(G) \rightarrow\{1,2,3, \ldots, k\}$ is called inclusive lucky number of a graph $G$ and it is denoted by $\eta_{\mathrm{i}}(G)$.

A labeling $\mathrm{f}$ is said to be proper inclusive lucky labeling if $S[u] \neq S[v]$ and $f(u) \neq f(v)$ for every pair of adjacent vertices $u$ and $v$. The least positive integer $k$ for which there exists a proper inclusive lucky labeling $f: V(G) \rightarrow\{1,2,3, \ldots, k\}$ is called proper inclusive lucky number of a graph $G$ and it is denoted by $\eta_{i p}(G)$.

A graph which admits an inclusive lucky labeling is called ILL graph and a graph which admits a proper inclusive lucky labeling is called PILL graph.

Theorem 1: For $\mathrm{n} \geq 2$, the complete graph $K_{n}$ is not ILL graph and it not PILL graph.

Proof: In a complete graph any two vertices are adjacent and so for any vertex $v, N[v]=V\left(K_{n}\right)$, the vertex set of $K_{n}$.

Therefore for any labeling $f, \quad S[v]$ is same for all vertices $v$. Thus there is no inclusive lucky labeling and proper inclusive lucking labeling for $K_{n}$ and hence $K_{n}$ is not an ILL graph and also not a PILL graph.

Remark: The complete graphs are lucky but not inclusive lucky. This shows that the lucky labeling and inclusive lucky labeling are different.

Theorem 2: For the path graph $P_{3}, \eta_{i}\left(P_{3}\right)=1$ and $\eta_{i p}\left(P_{3}\right)=2$.

Proof: Let the vertices of $P_{3}$ be $v_{1}, v_{2}$ and $v_{3}$ and let $v_{2}$ be the internal vertex. Then $\operatorname{deg}\left(v_{2}\right)=2$.

Label all vertices with 1 . Then $S\left[v_{1}\right]=2, S\left[v_{2}\right]=3$ and $S\left[v_{3}\right]=2$. Cleary for any two adjacent vertices $u$ and $v$ in $P_{3}, S[u] \neq S[v]$. Therefore, $f: V(G) \rightarrow\{1,2\}$ is an inclusive lucky labeling of $G$ and so $P_{3}$ is a ILL graph and $\eta_{i}\left(P_{3}\right)=1$.

The inclusive labeling defined above is not proper and so $\eta_{i p}\left(P_{3}\right) \geq 2$. Label the internal vertex as 2 and the end vertices with 1 , we get a proper inclusive lucky labeling 2 labels. Therefore, $P_{3}$ is a PILL graph and $\eta_{i p}\left(P_{3}\right)=$ 2 .

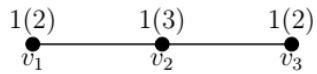

(a) Inclusive lucky labelling of $P_{3}$

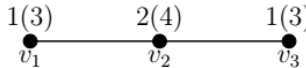

(b) Proper inclusive lucky labelling of $P_{3}$

Theorem 3: For $P_{n}(n>3), \eta_{i}\left(P_{n}\right)=2$ and $\eta_{i p}\left(P_{n}\right)=2$.

Proof: For $n>3, P_{n}$ has atleast two internal vertices which are adjacent. If we assign the number 1 to all vertices then $S[u]=3$ for all internal vertices and so for adjacent internal vertices $v$ and $w, S[v]=S[w]$. Therefore, we cannot give inclusive lucky labeling with $\{1\}$.

Consider the labels $\{1,2\}$. If we alternatively assign the labels 1 and 2 to the vertices of $P_{n}$, then we get a labeling which is inclusive and proper and so $P_{n}$ is ILL graph and PILL graph. Further $\eta_{i}\left(P_{n}\right)=\eta_{i p}\left(P_{n}\right)=2$.

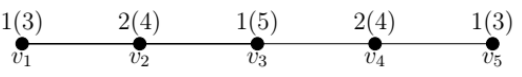

Inclusive lucky labelling of $P_{5}$

Definition: (Star graph $S_{n}$ ) A star graph $S_{n}$ is a graph obtained by joining n pendent edges to a single vertex. It has $n+l$ vertices and $n$ edges.

Theorem 4: For a star graph $S_{n}(\mathrm{n} \geq 2), \eta_{i}\left(\mathrm{~S}_{\mathrm{n}}\right)=1$ and $\eta_{i p}\left(\mathrm{~S}_{\mathrm{n}}\right)=2$.

Proof: Let $\left\{\mathrm{u}, v_{1}, v_{2}, \ldots, v_{\mathrm{n}}\right\}$ be the vertices of $\mathrm{S}_{\mathrm{n}}$ such that $u$ is a vertex of degree $n$ and each $v_{i}$ is a pendent vertex. Then $\left\{v_{1}, v_{2}, \ldots, v_{\mathrm{n}}\right\}$ is an independent set of vertices.

Label all the vertices of $S_{n}$ by 1 . Then it gives a labeling $f: V\left(S_{n}\right) \rightarrow\{1\}$ given by $f(v)=1$ for all vertices $v$ in $G$.

Note that $S\left[v_{i}\right]=2$ for $1 \leq i \leq n$ and $S[u]=n+1$.

Thus $S[a] \neq S[b]$ for any two adjacent vertices $a$ and $b$ in $S_{n}$ and so $f$ is an inclusive lucky labeling. Since only one label is used in this labeling, we have $\eta_{i}\left(S_{n}\right)=1$.

Further, this labeling $f$ is not proper.

Consider $g: V\left(S_{n}\right) \rightarrow\{1,2\}$ given by $g\left(v_{i}\right)=1$ for $1 \leq i \leq n$ and $g(u)=2$. Then clearly $g$ is proper and in this labeling, we have $S\left[v_{i}\right]=3$ for each $i$ and $S[u]=n+2$ implies that $g$ is inclusive lucky labeling. Thus $g$ is a proper inclusive lucky labeling and hence $\eta_{i p}\left(S_{n}\right)=2$.

Definition: (Bistar graph $\boldsymbol{B}_{n, m}$ ) A bistar graph $B_{n, m}$ is the graph obtained from $K_{2}$ by joining n pendant edges to one end and m pendant edges to the other end of $K_{2}$. 
Theorem 5: For a bistar graph $B_{n, m}$

$$
\eta_{i}\left(B_{n, m}\right)= \begin{cases}1 & \text { if } n \neq m \\ 2 & \text { it } n=m\end{cases}
$$

Proof: Let $u$ and $v$ be the vertices of degree $n$ and $m$ respective in $B_{n, m}$. Let $\left\{u_{1}, u_{2}, \ldots, u_{n}\right\}$ be the $n$ pendent vertices adjacent with $u$ and $\left\{v_{1}, v_{2}, \ldots, v_{m}\right\}$ be the $m$ pendent vertices adjacent with $v$.

Case (i): $n=m$.

Since $n=m$, both $u$ and $v$ have same degree and they are adjacent. If we label the vertices with $\{1\}$ then $S[u]$ $=S[v]$ and so such labeling is not an inclusive lucky labeling.

Consider $f: V\left(\mathrm{~B}_{\mathrm{n}, \mathrm{m}}\right) \rightarrow\{1,2\}$ given by

$$
\begin{gathered}
f(u)=1, f(v)=2, \\
f\left(u_{i}\right)=2 \text { for } 1 \leq i \leq n \text { and } f\left(v_{j}\right)=1 \text { for } 1 \leq j \leq m \\
+3=n+3, \\
S\left[u_{i}\right]=3 \text { for } 1 \leq i \leq n \text { and } S\left[v_{j}\right]=3 \text { for } 1 \leq j \leq m .
\end{gathered}
$$

Then $S[u]=2 n+3, S[v]=m+3=n+3$,

Therefore, $S[a] \neq S[b]$ for any two adjacent vertices $a$ and $b$ in $B_{n, m}$.

Thus $f$ is an inclusive lucky labeling and so $\eta_{i}\left(B_{n, m}\right)=2$.

Case (ii) $n \neq m$.

Label all the vertices with 1 .

Then $S[u]=n+2, S[v]=m+2$,

$S\left[u_{i}\right]=2$ for $1 \leq i \leq n$ and $S\left[v_{j}\right]=2$ for $1 \leq j \leq m$.

Since $n \neq m, S[u] \neq S[v]$. Therefore, $S[a] \neq S[b]$ for any two adjacent vertices $a$ and $b$ in $B_{n, m}$. Thus $f$ is an inclusive lucky labeling and so $\eta_{i}\left(B_{n, m}\right)=1$.

Theorem 6: For a bistar graph $B_{n, m}$

$$
\eta_{i p}\left(B_{n, m}\right)=\left\{\begin{array}{ll}
2 & \text { if } m \neq 2 n \\
3 & \text { if } m=2 n
\end{array} .\right.
$$

Proof: Let $u$ and $v$ be the vertices of degree $n$ and $m$ respective in $\mathrm{B}_{\mathrm{n}, \mathrm{m}}$. Let $\left\{u_{1}, u_{2}, \ldots, u_{n}\right\}$ be the $n$ pendent vertices adjacent with $u$ and $\left\{v_{1}, v_{2}, \ldots, v_{m}\right\}$ be the $m$ pendent vertices adjacent with $v$.

With $\{1\}$, we cannot define proper inclusive lucky labeling for $\mathrm{B}_{\mathrm{n}, \mathrm{m}}$ and so we consider the labels $\{1,2\}$.

Case (i): $m \neq 2 n$.

Since $n=m$, both $u$ and $v$ have same degree and they are adjacent. If we label the vertices with $\{1\}$ then $S[u]$ $=S[v]$ and so such labeling is not an inclusive lucky labeling.

Consider $f: V\left(B_{n, m}\right) \rightarrow\{1,2\}$ given by

$$
\begin{gathered}
f(u)=1, f(v)=2, \\
f\left(u_{i}\right)=2 \text { for } 1 \leq i \leq n \text { and } f\left(v_{j}\right)=1 \text { for } 1 \leq j \leq m .
\end{gathered}
$$

Then $S[u]=2 n+3, S[v]=m+3=n+3$,

$$
S\left[u_{i}\right]=3 \text { for } 1 \leq i \leq n \text { and } S\left[v_{j}\right]=3 \text { for } 1 \leq j \leq m .
$$

Since $m \neq 2 n, \mathrm{~S}[u] \neq S[v]$. Therefore, $S[a] \neq S[b]$ for any two adjacent vertices $a$ and $b$ in $B_{n, m}$.

Clearly $f$ is a proper. Therefore, $f$ is a proper inclusive lucky labeling and so $\eta_{i p}\left(B_{n, m}\right)=2$.

Case (ii) $m=2 n$.

Consider g: $V\left(B_{n, m}\right) \rightarrow\{1,2,3\}$ given by

$$
g(u)=2, g(v)=3 \text {, }
$$

Then $S[u]=n+5, S[v]=m+5$

$$
g\left(u_{i}\right)=1 \text { for } 1 \leq i \leq n \text { and } g\left(v_{j}\right)=1 \text { for } 1 \leq j \leq m .
$$

$S\left[u_{i}\right]=3$ for $1 \leq i \leq n$ and $S\left[v_{j}\right]=4$ for $1 \leq j \leq m$.

Since $m=2 n$, we have $S[u] \neq S[v]$. Therefore, $S[a] \neq S[b]$ for any two adjacent vertices $a$ and $b$ in $B_{n, m}$. Thus $g$ is a proper inclusive lucky labeling and so $\eta_{i p}\left(B_{n, m}\right)=3$.

Definition: (Cycle $\boldsymbol{C}_{\boldsymbol{n}}$ ) A cycle graph $C_{n}$ is a 2-regular simple graph with $n$ vertices and $n$ edges

Theorem 7: For even cycles $C_{2 n}(n \geq 2)$,

Proof: Let $C_{2 n}: v_{1}-v_{2}-\cdots-v_{2 n}-v_{1}$.

$$
\eta_{i}\left(C_{2 n}\right)=\eta_{i p}\left(C_{2 n}\right)=2
$$

For $n \geq 2, C_{2 n}$ has atleast two internal vertices and so an inclusive lucky labeling with $\{1\}$ is not possible.

$$
\text { Consider the labels }\{1,2\} \text {. Define } f: V\left(C_{2 n}\right) \rightarrow\{1,2\} \text { by } f\left(v_{i}\right)= \begin{cases}1 & \text { if } i \text { is odd } \\ 2 & \text { if } i \text { is even. }\end{cases}
$$

Then $f(u) \neq f(v)$ for any two adjacent vertices $u$ and $v$. Therefore, for this labeling $f$, we get

$$
S\left[v_{i}\right]= \begin{cases}5 & \text { if } i \text { is odd } \\ 4 & \text { if } i \text { is even }\end{cases}
$$


Note that $S[u] \neq S[v]$ for any two adjacent vertices $u$ and $v$. Thus $f$ is an inclusive lucky labeling of $C_{2 n}$ and so $\eta_{i}\left(C_{2 n}\right)=2$ Further this $f$ is proper and so $\eta_{i p}\left(C_{2 n}\right)=2$.

Theorem 8: For odd cycles $C_{2 n+1}(n \geq 2)$,

Proof: Let $C_{2 n+1}: v_{1}-v_{2}-\cdots-v_{2 n+1}-v_{1}$.

$$
\eta_{i}\left(C_{2 n+1}\right)=3 \text {. }
$$

For $n \geq 2, C_{2 n+1}$ has atleast two internal vertices and so an inclusive lucky labeling with $\{1\}$ is not possible.

Any labeling of $C_{2 n+1}$ with $\{1,2\}$ contains two adjacent vertices $u$ and $v$ such that $S[u]=\mathrm{S}[v]$. Therefore, such labeling is not an inclusive lucky labeling and so $\eta_{i}\left(C_{2 n+1}\right) \geq 3$.

We consider the following three cases.

Case (1): $2 n+1$ is divisible by 3 .

Then $2 n+1=3 m$ where $m$ is odd.

Subcase (i) Let $m \equiv 0(\bmod 3)$ or $m \equiv 2(\bmod 3)$.

Define $f: V\left(C_{2 n+1}\right) \rightarrow\{1,2,3\}$ by

$$
f\left(v_{i}\right)=\left\{\begin{array}{lll}
1 & \text { if } i \equiv 1,2,3 & (\bmod 9) \\
2 & \text { if } i \equiv 4,5,6 & (\bmod 9) \\
3 & \text { if } i \equiv 7,8,9 & (\bmod 9)
\end{array}\right.
$$

For this labeling $f$, the possible value of $S[v]$ are $\{3,4,5,6,7,8,9\}$. Further $S\left[v_{i}\right] \neq S\left[v_{i+1}\right]$ and $S\left[v_{i}\right] \neq S\left[v_{i-1}\right]$ for any $i$. Therefore, $S[u] \neq S[v]$ for any two adjacent vertices $u$ and $v$ in $\mathrm{C}_{2 n+1}$. Thus $f$ is an inclusive lucky labeling.

Subcase (ii) Let $m \equiv 1(\bmod 3)$.

Define $f: V\left(C_{2 n+1}\right) \rightarrow\{1,2,3\}$ by

$$
\begin{gathered}
f\left(v_{1}\right)=f\left(v_{2}\right)=f\left(v_{3}\right)=1, \\
f\left(v_{n}\right)=f\left(v_{n-1}\right)=f\left(v_{n-2}\right)=2
\end{gathered}
$$

and for the remaining $i$

$$
f\left(v_{i}\right)=\left\{\begin{array}{lll}
1 & \text { if } i \equiv 1,2,3 & (\bmod 9) \\
2 & \text { if } i \equiv 4,5,6 & (\bmod 9) \\
3 & \text { if } i \equiv 7,8,9 & (\bmod 9)
\end{array}\right.
$$

In this labeling $f, S[u] \neq S[v]$ for any two adjacent vertices $u$ and $v$ in $C_{2 n+1}$. Thus $f$ is an inclusive lucky labeling. Case(2): $2 n+1$ is not divisible by 3 .

Define $f: V\left(C_{2 n+1}\right) \rightarrow\{1,2,3\}$ by

$$
f\left(v_{i}\right)=\left\{\begin{array}{lll}
1 & \text { if } i \equiv 1,2 & (\bmod 6) \\
2 & \text { if } i \equiv 3,4 & (\bmod 6) \\
3 & \text { if } i \equiv 5,6 & (\bmod 6) .
\end{array}\right.
$$

Note that $S[u] \neq S[v]$ for any two adjacent vertices $u$ and $v$. Thus $f$ is an inclusive lucky labeling of $C_{2 n+1}$.

Hence $\eta_{i}\left(C_{2 n+1}\right)=3$.

Theorem 9: For odd cycles $C_{2 n+1}(n \geq 3), \eta_{i p}\left(C_{2 n+1}\right)=4$ and $\eta_{i p}\left(C_{5}\right)=5$.

Proof: We know that for $\chi\left(C_{2 n+1}\right)=3$. Since $\eta_{i p}(G) \geq \chi(G)$, we have $\eta_{i p}\left(C_{2 n+1}\right) \geq 3$.

For any proper labeling of $C_{2 n+1}$ with $\{1,2,3\}$ contains two adjacent vertices $u$ and $v$ such that $S[u]=\mathrm{S}[v]$. Therefore, there exists no proper inclusive lucky labeling of $C_{2 n+1}$ with $\{1,2,3\}$ and so $\eta_{i p}\left(C_{2 n+1}\right) \geq 4$.

Note that there is no proper inclusive lucky labeling for $C_{5}$ with 4 labels and so $\eta_{i p}\left(C_{5}\right)=5$.

Define $f: V\left(C_{2 n+1}\right) \rightarrow\{1,2,3,4\}$ by

$$
\begin{array}{r}
f\left(v_{1}\right)=1, f\left(v_{2}\right)=2, f\left(v_{n}\right)=2 \text { and } \text { for } 3 \leq i \leq n-1 \\
f\left(v_{i}\right)=\left\{\begin{array}{lll}
3 & \text { if } i \equiv 1 & (\bmod 2) \\
4 & \text { if } i \equiv 0 & (\bmod 2)
\end{array}\right.
\end{array}
$$

Then clearly $S\left[v_{1}\right]=5, S\left[v_{2}\right]=6, S\left[v_{3}\right]==S\left[v_{n-1}\right], S\left[v_{n}\right]=7$ and for $4 \leq i \leq n-2$,

$$
S\left[v_{i}\right]=\left\{\begin{array}{lll}
10 & \text { if } i \equiv 0 & (\bmod 2) \\
11 & \text { if } i \equiv 1 & (\bmod 2)
\end{array}\right.
$$

Thus $f$ is a proper inclusive lucky labelling of $C_{2 n+1}$ and hence $\eta_{i p}\left(C_{2 n+1}\right)=4$.

Definition: (Complete Bipartitie $\boldsymbol{K}_{n, \boldsymbol{m}}$ ). A complete bipartite graph is agraph whose vertex set $\mathrm{V}$ can be partitioned into two subsets $V_{1}$ and $V_{2}$ (i.e., $V=V_{1} U V_{2}$ and $V_{1} \cap V_{2}=\phi$ ) such that the edge set $E=V_{1} \times V_{2}$.It is denoted by $K_{n, m}$ where $\mathrm{n}=\left|\mathrm{V}_{1}\right|$ and $\mathrm{m}=\left|\mathrm{V}_{2}\right|$. 
Theorem 10: For a complete bipartite graph $K_{n, m}(n, m \geq 2)$

Further $\eta_{i p}\left(K_{n, m}\right)=2$ for all $n$ and $m$.

$$
\eta_{i}\left(K_{n, m}\right)= \begin{cases}1 & \text { if } m \neq n \\ 2 & \text { if } m=n\end{cases}
$$

Proof: Let $\mathrm{V}_{1}$ and $\mathrm{V}_{2}$ be the bipartition of the vertex set $\mathrm{V}$ and let $\mathrm{V}_{1}=\left\{u_{1}, u_{2}, \ldots, u_{n}\right\}$ and $\mathrm{V}_{2}=\left\{v_{1}, v_{2}, \ldots, v_{m}\right\}$. Case(i): $n=m$.

If we label all the vertices with $\{1\}$ then $S[v]=\mathrm{n}+1$ for any vertex $v$ and so such labeling is not an inclusive lucky labeling.

Consider $f: V\left(K_{n, n}\right) \rightarrow\{1,2\}$ given by

$$
f\left(u_{i}\right)=1 \text { for } 1 \leq i \leq n \text { and } f\left(v_{j}\right)=2 \text { for } 1 \leq j \leq n .
$$

Then $S\left[u_{i}\right]=2 n+1$ for $1 \leq i \leq n$ and $S\left[v_{j}\right]=n+2$ for $1 \leq j \leq n$.

Therefore, $S[a] \neq S[b]$ for any two adjacent vertices $a$ and $b$ in $K_{n, n}$.

Thus $f$ is an inclusive lucky labeling and so $\eta_{i}\left(K_{n, n}\right)=2$.

Note that $f$ is proper also. Thus $f$ is a proper inclusive lucky labeling and hence $\eta_{i p}\left(K_{n, n}\right)=2$.

Case (ii) $n \neq m$.

Label all the vertices with 1 . Then $S\left[u_{i}\right]=n+l$ for $1 \leq i \leq n$ and $S\left[v_{j}\right]=m+l$ for $1 \leq j \leq m$.

Since $n \neq m, S[a] \neq S[b]$ for any two adjacent vertices $a$ and $b$ in $K_{n, m}$.

Thus $f$ is an inclusive lucky labeling and so $\eta_{i}\left(K_{n, m}\right)=1$.

Further such labeling is not proper, so we consider the labeling $f$ as in case (i).

Without loss of generality we may assume $m>n$.

Define $f: V\left(K_{n, m}\right) \rightarrow\{1,2\}$ given by

Then $S\left[u_{i}\right]=2 m+1$ for $1 \leq i \leq n$ and

$$
f\left(u_{i}\right)=1 \text { for } 1 \leq i \leq n \text { and } f\left(v_{j}\right)=2 \text { for } 1 \leq j \leq m .
$$

$S\left[v_{j}\right]=n+2$ for $1 \leq j \leq m$.

Note that $2 m+1=n+2$ implies $\mathrm{m}<\mathrm{n}$.

Since $m>n, 2 m+1 \neq n+2$ and $S[a] \neq S[b]$ for any two adjacent vertices $a$ and $b$ in $K_{n, m}$.

Thus $f$ is a proper inclusive lucky labeling of $K_{n, m}$ and hence $\eta_{i p}\left(K_{n, m}\right)=2$.

\section{CONCLUSION}

Different notion of labeling are defined in graphs and they are widely used in different areas viz coding theory, communication network. In this article, we defined a new notion of labeling called inclusive lucky labeling

and discussed this labeling on some standard graphs.

\section{REFERENCES}

1. A. Ahadi, A. Dehghan, M. Kazemi, E. Mollaahmedi, "Computation of Lucky number of planar graphs in NP-hard”, Information Processing Letters, Vol. 112 (4), pp. 109-112, 15 Feb 2012.

2. S. Akbari, M. Ghanbari, R. Manariyat, S. Zare, "On the lucky choice number of graphs", Graphs and Combinatorics, Vol. 29 n.2, pp. 157-163, Mar 2013.

3. R. L. Graham and N.J.A. Sloane, "On additive bases and harmonious graphs", SIAM J. Alg. Disc. Meth, Vol. 1, pp. 382 - 404, 1980.

4. A. Rosa, "On certain valuations of the vertices of a graph", Theory of Graphs (Inter-nat. symposium, Rome), pp. 349 - 355, 1967. 